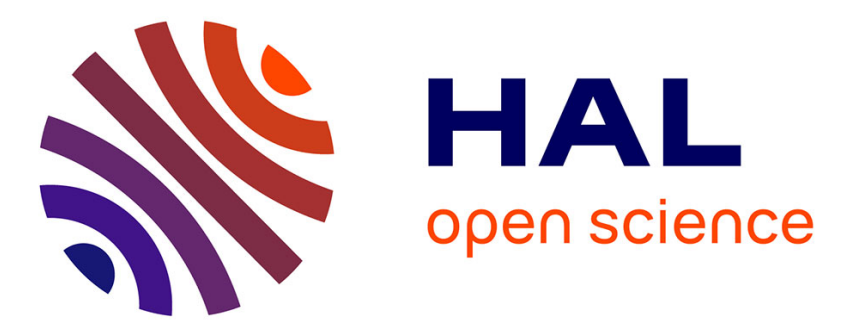

\title{
Les déterminants du risque perçu par le touriste français lors du choix d'une destination touristique: un modèle conceptuel
}

\author{
Rima Yousfi-Bachir, Johannes Schaaper
}

\section{- To cite this version:}

Rima Yousfi-Bachir, Johannes Schaaper. Les déterminants du risque perçu par le touriste français lors du choix d'une destination touristique: un modèle conceptuel. Question(s) de Management, 2017, Le risque en question(s), 16 (1), pp.49-59. 10.3917/qdm.171.0049 . hal-03258207v2

\section{HAL Id: hal-03258207 \\ https://hal.science/hal-03258207v2}

Submitted on 5 Jul 2021

HAL is a multi-disciplinary open access archive for the deposit and dissemination of scientific research documents, whether they are published or not. The documents may come from teaching and research institutions in France or abroad, or from public or private research centers.
L'archive ouverte pluridisciplinaire HAL, est destinée au dépôt et à la diffusion de documents scientifiques de niveau recherche, publiés ou non, émanant des établissements d'enseignement et de recherche français ou étrangers, des laboratoires publics ou privés. 


\title{
Les déterminants du risque perçu par le touriste français lors du choix d'une destination touristique : un modèle conceptuel
}

\author{
The determinants of perceived risk when choosing a tourist \\ destination:
}

Rima YOUSFI-BACHIR et Jan SCHAAPER

\begin{abstract}
Résumé
L'actualité géopolitique et des évènements marquants médiatisés (attentats, catastrophes, crash...) de ces dernières années amènent à reconsidérer la question du risque dans le champ touristique. Aujourd'hui, la perception du risque menace plus que sa réalité. Cet article propose de déterminer les facteurs subjectifs à l'origine du risque perçu, lors du choix d'une destination touristique. Pour répondre à cette problématique, sur la base d'une revue de littérature académique, nous avons interrogé 16 professionnels du tourisme en face-à-face. Nous trouvons neuf facteurs subjectifs à l'origine de la perception du risque dans le choix d'une destination touristique, qui se regroupent en trois thématiques : le profil psychographique du touriste, les caractéristiques perçues de la destination et la situation subjective du choix. Aussi, nous trouvons que parmi les huit dimensions théoriques du risque perçu, c'est la dimension physique qui est prépondérant dans le tourisme. Notre recherche aboutit sur un modèle conceptuel de la perception du risque dans le tourisme.
\end{abstract}

Mots-clefs : risque perçu, tourisme, comportement du consommateur, approche subjective, origine multidimensionnelle, actualité géopolitique, risque physique.

\section{Summary}

The current geopolitical situation and marking events, highlighted by media coverage (attacks, catastrophes, crashes...) of recent years, lead to reconsider the question of risk in the field of tourism. Today, the perception of risk threatens more than its reality. The purpose of this research is to identify the subjective determinants of the perceived risk, when choosing a tourist destination. To answer this question, on the basis of a review of academic literature, we interviewed 16 tourism professionals in a face-to-face setting. We find that nine subjective factors, or antecedents, are at the origin of risk perception when a tourist chooses his destination. These antecedents can be gathered into three themes: the tourist's psychographic profile, the perceived characteristics of the destination and the subjective situation. Also, we find that among the eight theoretical dimensions of perceived risk, the physical dimension is preponderant in tourism. Our research leads to a conceptual model of risk perception in tourism.

Keywords: perceived risk, tourism, consumer behavior, subjective approach, multi-dimensional antecedent, geopolitical situation, physical risk. 


\section{INTRODUCTION}

Le tourisme est aujourd'hui plus que jamais marqué par une dimension internationale. La tendance est aux déplacements lointains, fréquents et de courte durée (rapport d'études Raffour, 2013). Le nombre de touristes a attaint 1.2 milliard en 2015, soit une hausse de $4 \%$ par rapport à l'année 2014 (OMT, 2016). À I'horizon de 2030, 1,8 milliard de touristes internationaux sont attendus dans le monde.

Étant directement liée à la situation géopolitique mondiale, cette croissance du tourisme va de pair avec la montée de l'insécurité et de la peur. À travers les médias, nous assistons à divers problèmes et menaces. Des mouvements sociaux au Moyen-Orient et au Maghreb (printemps arabe, 2010-2013), des attentats terroristes (musée du Bardo et Hôtel Imperial Marhaba en Tunisie, 2015), des accidents de transport aérien (crash de la Germanwings, 2014), des crises sanitaires (le virus Ebola, 2014) et des catastrophes naturelles (séisme au Népal, 2015), sans oublier les attentats en France en 2015 et 2016. Tout cela n'est pas sans conséquence sur la filière touristique.

Alors que "la sécurité est un facteur indispensable pour poursuivre le développement des activités touristiques" (Denécé et Meyer, 2006, p.120), on assiste depuis le 11 septembre 2001 à un redéploiement massif de la question du risque dans le domaine du tourisme (Korstanje, 2011). La destination touristique renvoie à un imaginaire puissant et renferme une grande part d'affectif. $\mathrm{Ce}$ sont les perceptions et les interprétations mentales individuelles plus que les réalités qui déterminent le choix du touriste (Roehl et Fesenmaier, 1992). Le risque perçu est un élément qui conditionne l'intérêt porté à une destination ou bien l'évitement de celle-ci (Sönmez et Graefe, 1988). Dans ses travaux sur le tourisme des Français en Égypte, Tare Sayed (2010) souligne que le nombre de touristes baisse lorsque le risque perçu associé à une destination touristique croît.

"Les divers facteurs qui favorisent, voire engendrent la perception d'un risque avant l'achat peuvent être liés au consommateur et au produit, mais aussi à la situation d'achat » (Volle 1995, p.12). Cependant, la portée des études publiées sur les variables à l'origine du risque perçu ne concerne que des situations de choix générales. Bien qu'un nombre important de déterminants a été identifié, force est de constater que dans le cas particulier du choix de la destination touristique, nous ne connaissons pas les véritables causes du risque perçu.

L'objectif de cette étude est d'enrichir la connaissance sur le risque perçu en clarifiant ses principaux déterminants subjectifs dans le contexte d'un touriste français. Le choix d'une destination touristique revêt un caractère distinctif par rapport à d'autres actes de consommation, car il est impossible d'évaluer le produit en amont. Cette part d'inconnu explique les appréhensions du touriste. Partir en voyage touristique implique deux situations contradictoires, d'autant plus confirmées ces dernières années, entre la recherche du plaisir et l'exposition à plusieurs risques. Ces derniers sont perçus différemment d'un touriste à l'autre.

Dans la présente étude, nous exposons le bilan de la recherche dans la littérature académique sur le risque perçu et les trois positions théoriques que nous avons retenues. Ensuite, nous expliquons la méthodologie de la construction d'un modèle conceptuel, sur la base conjointe de la littérature et de seize entretiens semi-directifs menés avec les professionnels du tourisme. Enfin, nous discutons les résultats en passant en revue neuf facteurs à l'origine du risque perçu dans le choix d'une destination touristique, et concluons avec les principaux apports de notre recherche.

\section{Le cadre théorique et conceptuel}

Afin de créer un cadre d'analyse des facteurs subjectifs pouvant influencer le risque perçu, nous avons rompu avec la vision traditionaliste selon laquelle le risque est un ensemble de probabilités rationnelles (Calendre, 2006). Nous avons privilégié les considérations psychologiques du risque perçu (Bauer, 1960 ; Volle, 1995 ; Mallet, 2004). En adoptant le modèle tripartite de Volle (1995) repris par Mallet (2004), nous étudions la triple origine du risque perçu. Selon ces auteurs, le risque perçu est le résultat de l'interaction simultanée entre un individu, un produit et une situation 
d'achat (Mallet, 2004). Enfin, en accord avec les conclusions de (Volle, 1995) puis Mitchell et Vassos (1997) sur la multidimensionnalité du risque perçu, nous considérons les huit dimensions identifiées dans le tourisme (Korstanje, 2011 ; Croutsche et Roux, 2005) et nous nous interrogeons sur l'importance de chacune dans la perception du risque global.

\subsection{Le risque perçu, un phénomène subjectif}

L'une des premières définitions du risque perçu a été initiée par Bauer en 1960. II affirme que « le comportement du consommateur implique un risque dans le sens où chaque achat va engendrer des conséquences, parfois négatives, qu'il ne peut anticiper avec certitude " (Volle, 1995, p1). Plusieurs auteurs en sciences de gestion ont défini la portée et le sens du risque perçu. C'est un phénomène dont les limites ne sont pas clairement définies (Brunel et Pichon, 2002). Certains sont unanimes sur son caractère subjectif et intime comme Ayadi (2016). Volle (1995, p.9) le définit comme " l'incertitude subjectivement perçue par un individu quant à l'ensemble des pertes potentielles relatives aux attributs déterminants du choix d'un produit (bien ou service) dans une situation d'achat ou de consommation donnée ».

Le risque perçu est décomposé en deux élé-

ments : «l'incertitude et les conséquences négatives ". Cette décomposition s'est confirmée au travers de plusieurs recherches (Taylor, 1974 ; Peter et Ryan, 1976 ; Volle, 1995). L'incertitude dans le risque perçu est liée à un processus de jugement psychologique, quant à la fiabilité du choix de l'individu. Selon Cunningham (1967), le degré de l'incertitude associé à chaque perte (conséquence négative) est subjectif. Ainsi, la probabilité d'une même perte (conséquence négative) peut être appréciée différemment par deux personnes. La conséquence négative (la perte) « caractérise la situation où un individu obtient un résultat inférieur à un point de référence »(Volle, 1995, p.4). Le point de référence n'est autre que le résultat du vécu du consommateur, de son environnement et de ses croyances.

\subsection{Le risque perçu, un concept multidimensionnel}

Bauer (1960) a étudié le risque perçu au travers de cinq dimensions : «le risque financier, le risque de performance, le risque physique, le risque psychosocial et le risque global ». Mitchell et Vassos (1997) mettent en avant une nouvelle façon d'appréhender le risque perçu en le décomposant en plusieurs attributs «modèle multi-attributs ». Ce modèle ne réduit pas le risque perçu à quelques dimensions, il s'adapte au contexte du choix, au type de produit ou service pour lesquels l'individu perçoit un risque. Selon ce modèle, il y aurait pour chaque attribut du produit ou du service une probabilité de risque perçu. Stone et Grônhaug (1993) évaluent l'impact de chacune des dimensions du risque perçu sur la perception du risque global. D'après leurs résultats, ce sont les conséquences négatives psychologiques et financières du choix d'un produit ou service, qui contribuent fortement au risque perçu.

Dans le choix d'une destination touristique, les chercheurs identifient huit dimensions de risque perçu (Sonmez, 1998 ; Lepp et Gibson, 2003) : le risque de terrorisme, le risque d'instabilité politique, le risque sanitaire, le risque de crime, le risque économique, le risque culturel, les dogmes religieux et le risque de catastrophes naturelles. Croutsche et Roux (2005) ont classifié ces dimensions selon le niveau d'inquiétude qu'elles suscitent chez le touriste. Les risques liés à l'instabilité politique, au terrorisme et à la santé physique se voient attribuer le premier rang des risques ayant une influence significative sur le choix du touriste. Suivis par les risques de crime, notamment ceux liés aux enlèvements, aux vols, aux viols, aux risques de transports liés aux accidents et aux détournements d'avions. Enfin, les risques économiques en lien avec le fonctionnement des institutions financières influencent en moindre proportion le choixdu touriste. Le risque de catastrophes naturelles n'est pas considéré comme étant fondamental. «En d'autres termes, les touristes ne voient pas d'inconvénients majeurs dans ce type de situation ou ne se sentent pas concernés en priorité par ce type de risques » (Croutsche et Roux, 2005, p.66). Par ailleurs, les risques de barrières culturelles et les dogmes religieux sont considérés comme étant des risquessecondaires. 


\subsection{Le risque perçu, une origine multi-source}

Les études sur les déterminants du risque perçu avant l'achat révèlent de nombreuses variables. Celles-ci appartiennent à trois origines différentes : « les caractéristiques du consommateur, du produit et de la situation d'achat » (Chaudhuri, 1998 ; Volle, 1995 ; Mallet, 2004. Selon Volle, 1995), c'est l'interaction simultanée entre ces trois facteurs qui crée le risque perçu. En accord avec la thèse du caractère perceptuel du risque (Ayadi, 2006), nous nous intéressons dans cette recherche à l'étude des déterminants subjectifs supposés être à l'origine du risque perçu.

Les caractéristiques individuelles influencent le risque perçu. Quatre traits de la personnalité ont fait l'objet de conclusions contradictoires : la confiance en soi spécifique (Cunningham, 1967, Schaninger, 1976), la sensibilité au risque (Sjöberg, 2000), la sensibilité au risque spécifique (Mallet, 2004) et l'anxiété (Schaninger, 1976). Le style cognitif est le seul trait de la personnalité à l'origine du risque perçu qui a suscité une position unanime par les chercheurs (Raju, 1980). Par ailleurs, le pouvoir prédictif de la culture du consommateur sur le risque perçu a été testé et validé (Verhage, Yavas et Green, 1991 ; Rippl, 2002).

Comme l'a souligné Mallet (2004, p.3), « le risque perçu dépend non seulement du type de produit considéré, mais aussi de la façon dont le consommateur le perçoit ». En effet, le produit ou le service renferme un ensemble d'attributs abstraits, perçu différemment d'un individu à l'autre. Une corrélation positive a été confirmée entre la nouveauté perçue et le risque perçu (Popielarz, 1967 ; Roehrich, 1987). Par ailleurs, Mallet (2004) démontre l'influence de la déception à l'égard des achats antérieurs sur le risque perçu. Selon sa vision, ce n'est pas l'expérience accumulée (Cases, 2001) qui influence le risque perçu, mais c'est l'expérience négative qui le génère.

La situation subjective d'achat est un facteur important dans l'émergence du risque perçu. Mallet (2004) constate le manque de recherches qui s'intéressent à cette variable et l'approche objective du peu de recherches qui existe. II affirme que « le construit du risque perçu à l'égard de l'achat d'un produit est davantage de nature situationnelle » (Mallet, 2004, p.5). II souligne l'influence de trois caractéristiques de la situation d'achat sur l'émergence du risque perçu : la difficulté perçue du choix, l'importance perçue de la dépense et les réactions affectives négatives suscitées par le choix comme le souci, l'anxiété, la nervosité et le stress (Chaudhuri, 1998). En somme, le risque perçu dans le tourisme est généré par les traits de la personnalité et le profil psychologique du touriste, les caractéristiques perçues de la destination touristique et la situation subjective du choix de la destination touristique.

Le but de notre recherche est de répondre à la question suivante : quels sont les facteurs subjectifs à l'origine du risque perçu lors du choix d'une destination touristique ? Pour répondre à ce questionnement, nous avons élaboré un modèle conceptuel sur la base de la revue de littérature en gestion et en psychologie descriptive du consommateur, d'une part, et d'une étude qualitative exploratoire, que nous avons menée auprès des professionnels du tourisme, d'autre part. Ultérieurement, lors d'une deuxième phase, nous souhaitons confirmer ce modèle à travers une enquête quantitative auprès d'un large panel de touristes français.

\section{La méthodologie de la recherche}

Les nombreux facteurs supposés à l'origine du risque perçu ont été validés dans différents contextes d'achat et environnements. Ils sont pour la plupart testés séparément avec une approche objective du risque (Mallet, 2004). Afin de ne pas nous appuyer uniquement sur la revue de la littérature, qui n'est pas toujours appropriée à notre terrain d'investigation, nous avons mené une étude qualitative auprès de 16 experts du tourisme. L'objectif d'interviewer les professionnels du tourisme est double. D'une part, cela permet d'avoir la vision des experts sur le ressenti du touriste avec qui ils sont en interaction permanente. D'autre part, c'est une étude préalable qui permettra de préciser l'étude auprès des consommateurs-touristes, qui suivra ultérieurement.

L'approche qualitative de notre question de recherche se justifie, d'une part, par l'absence des études sur les antécédents du risque perçu propres au choix d'une destination. D'autre part, les facteurs à l'origine du risque perçu, développés dans la littérature, nécessitent une discrimination des plus prépondérants pour le cas du tourisme. En d'autres termes, quels sont les facteurs qui 
jouent le plus sur le degré de risque perçu.

\subsection{La collecte de données}

Pour avoir les premiers éléments de réponse à la problématique des antécédents du risque perçu dans le tourisme, nous avons mené une étude qualitative auprès des professionnels du tourisme. La méthodologie retenue est une étude qualitative basée sur des entretiens semi-directifs individuels qui s'est déroulée entre janvier et juin 2014. Un échantillon de seize professionnels a été sélectionné avec une moyenne d'âge de trente-cinq ans. Chacun a une mission particulière dans l'élaboration de la stratégie touristique. Ainsi, le critère de la diversité des cas a été respecté. La durée moyenne des entretiens a été d'environ 45 minutes. Nous avons enregistré les discussions et pris des notes par mots-clefs. Les entretiens ont été retranscrits intégralement.

Les entretiens ont été menés à l'aide d'un guide d'entretien, structuré en trois phases : une première phase où l'on laisse l'interlocuteur s'exprimer librement sur la question du risque perçu et ses déterminants, une deuxième phase où l'on propose à l'interlocuteur les facteurs subjectifs à l'origine du risque perçu, identifiés dans la littérature, et on lui demande d'en sélectionner les plus prépondérants dans le cas du tourisme et, enfin, une dernière phase où l'on invite l'interlocuteur à justifier son choix de variables. Le tableau 1 présente la structure du guide d'entretien.

\subsection{L'analyse des entretiens}

Pour décrypter nos 16 entretiens semi-directifs, nous avons utilisé une grille d'analyse de contenu thématique. " II s'agit d'un effort d'interprétation qui se balance entre deux pôles, d'une part la rigueur de l'objectivité, et d'autre part, la fécondité de la subjectivité » (Bardin, 1977, p.9). L'objectif est d'avoir une représentation synthétique et une mise à plat du discours des personnes interrogées. Le contenu discursif des entretiens et les informations recueillies ont été retranscrits « manuellement » en respectant les interjections, les tonalités et les silences. Une lecture approfondie nous a permis de repérer les points essentiels qui composent chacun des seize entretiens. Le corpus a été découpé en unités de contenu (catégories). Les parties d'entrevues comparables ont été regroupées par thèmes ou par sous-thèmes et leur sens a été identifié. " Ce codage sert à identifier les dimensions clés des discours, soit en prolongement direct des thèmes du guide d'entretien, donc fondés sur la littérature académique, soit en émergence pure à partir des points abordés et des explications fournies par les répondants 》 (Easterby-Smith et al., 2008, p. 73).

La phase centrale de l'analyse consiste à l'exploitation du matériel de l'enquête grâce à l'élaboration d'une grille. Chaque ligne de cette grille représente un entretien et chaque colonne une des thématiques retenues, qui rassemble les éléments ayant un caractère commun sous un titre générique. Les propos discursifs des professionnels du tourisme sont résumés et réduits à des phrases synthétiques, ensuite ventilés sur les différentes cellules de la grille. 
Tableau 1 : Structure du guide d'entretien

\begin{tabular}{|c|c|}
\hline Séquence & Contenu \\
\hline Phase introductive & $\begin{array}{l}\text { Présentation de l'objet de l'étude et de ses } \\
\text { objectifs }\end{array}$ \\
\hline \multicolumn{2}{|c|}{ Phase 1 : Entretien semi-directif } \\
\hline Le tourisme dans le contexte actuel & $\begin{array}{l}\text { Faire parler les interlocuteurs sur les tendances } \\
\text { du marché touristique, la crise et les nouveaux } \\
\text { besoins, les comportements du touriste }\end{array}$ \\
\hline $\begin{array}{l}\text { Les risques perçus par le touriste (nature, type, } \\
\text { dimensions) }\end{array}$ & $\begin{array}{l}\text { Qu'est-ce que le risque dans le tourisme? Les } \\
\text { risques réels et les risques perçus }\end{array}$ \\
\hline $\begin{array}{l}\text { L'origine du risque perçu par le touriste lors du } \\
\text { choix d'une destination touristique }\end{array}$ & $\begin{array}{l}\text { Quels antécédents au risque perçu dans le } \\
\text { tourisme? Hiérarchiser les facteurs cités, par ordre } \\
\text { d'importance }\end{array}$ \\
\hline \multicolumn{2}{|c|}{ Phase 2 : Entretien semi-directif (assisté) } \\
\hline Les sources du risque perçu dans le tourisme & $\begin{array}{l}\text { Après la proposition des différents facteurs } \\
\text { identifiés dans la littérature, les professionnels } \\
\text { du tourisme choisissent ceux qui leur semblent } \\
\text { à l'origine du risque perçu dans le choix d'une } \\
\text { destination touristique }\end{array}$ \\
\hline \multicolumn{2}{|c|}{ Phase 3 : Entretien semi-directif (assisté poussé) } \\
\hline Les sources du risque perçu dans le tourisme & $\begin{array}{l}\text { Les professionnels du tourisme argumentent } \\
\text { leurs choix et précisent l'étendue de l'influence de } \\
\text { chaque identifiant choisi }\end{array}$ \\
\hline
\end{tabular}

Ainsi, à l'intersection d'une ligne et d'une colonne, se trouvent les propos d'un professionnel $X$ sur un thème $Y$. Nous avons ensuite procédé à une analyse verticale, colonne par colonne, thème par thème, à la recherche de similitudes et différences dans les 16 discours. Enfin, nous avons compté le nombre de mots-clefs et expressions en rapport avec les antécédents du risque perçu dans le tourisme. Cette analyse nous a permis d'identifier les principaux raisonnements des professionnels du tourisme.

\section{Les résultats de l'analyse des entretiens qualitatifs}

Conformément à notre problématique centrale de recherche, l'analyse des entretiens a eu pour objectif d'enrichir la connaissance sur le risque perçu lors du choix d'une destination touristique, et d'en identifier les principaux déterminants subjectifs. Cette section traite successivement du risque perçu dans le discours des enquêtés $(\$ 3.1)$, des facteurs à l'origine du risque perçu (\$3.2) et aboutit sur un modèle conceptuel des facteurs qui influencent le risque perçu dans le tourisme (§3.3). Nous en tirons la conclusion que parmi les huit dimensions du risque perçu, recensées dans la littérature, c'est la dimension physique qui a le plus d'importance pour les touristes.

\subsection{Le risque perçu dans le discours des enquêtés}

Les définitions du concept de risque perçu dans la littérature académique comportent deux éléments clefs « l'incertitude et les conséquences négatives d'un choix ». Le tableau 2 répertorie quelques verbatim de " l'incertitude » et des " conséquences négatives » relevés des entretiens semi-directifs.

Au cours des entretiens, les enquêtés évoquent avec une fréquence non négligeable un certain nombre de risques majeurs pour le touriste, notamment ceux liés à un préjudice physique comme 
la mort, l'accident ou la maladie. Cela se traduit par des expressions comme « le terrorisme », « l'enlèvement » et « les épidémies ».

\subsection{Les facteurs à l'origine du risque perçu}

En s'interrogeant sur les facteurs à l'origine du risque perçu dans le tourisme, les entretiens font émerger deux principales thématiques. Tout d'abord, il y a celle liée à la dimension financière de l'achat. Classiquement, l'individu accorde une importance au prix dans son choix. Un coût élevé pourrait le dissuader, notamment à cause de l'impossibilité d'évaluer le rapport qualité/ prix au moment de l'achat. Également classiquement, un prix bas est susceptible de faire douter le touriste sur l'attractivité de la destination et les services proposés. "Les petits prix sont générateurs de risque perçu et les prix élevés supposent un investissement risqué » (discours des enquêtés). La seconde thématique soulevée par les répondants regroupe plusieurs facteurs. D'abord, l'insécurité de la destination, qui est perçue différemment d'un touriste à l'autre. Elle est étroitement liée aux événements marqués par la violence dans les destinations touristiques ou dans les zones limitrophes. Ensuite, les professionnels du tourisme parlent des médias, notamment numériques (le web et les réseaux sociaux) comme étant un facteur déterminant dans la propagation du risque perçu.

Tableau 2 : Verbatim du discours relevant deux composantes du risque perçu

\begin{tabular}{|l|l|}
\hline \multicolumn{1}{|c|}{ Verbatim de « l'incertitude » du touriste } & \multicolumn{1}{c|}{$\begin{array}{l}\text { Verbatim des «conséquences négatives » } \\
\text { redoutées par le touriste }\end{array}$} \\
\hline $\begin{array}{l}\text { «Au fait, le touriste ne sait pas exactement à } \\
\text { quoi se fier ... à quelle information et à quel } \\
\text { intermédiaire du tourisme faire confiance » } \\
\begin{array}{l}\text { (enquêté 1) } \\
\text { «On développe tous les jours de nouvelles } \\
\text { formules touristiques ... on propose au client } \\
\text { un nombre incroyable de possibilités de séjour, } \\
\text { malheureusement cela crée une confusionet un } \\
\text { doute plus qu'autre chose » (enquêté 12) }\end{array}\end{array}$ & $\begin{array}{l}\text { « On peut se permettre de se tromper dans l'achat } \\
\text { de n'importe quel produit, mais faire un mauvais } \\
\text { choix et prendre l'avion pour une destination } \\
\text { dangereuse, peut avoir des conséquences } \\
\text { dramatiques sur le touriste. » (enquêté 12) } \\
\text { « Si l'on s'autorise un seul congé par an, ce n'est } \\
\text { sûrement pas pour le regretter ou gaspiller son } \\
\text { argent » (enquêté 16) }\end{array}$ \\
\hline
\end{tabular}

Les informations négatives, dont les avis des internautes relayés par Facebook, Tweeter ou Youtube contribuent à la transformation des perceptions à l'égard des destinations touristiques et à l'émergence du risque perçu.

L'analyse des entretiens a permis de faire une discrimination des déterminants subjectifs du risque perçu identifiés dans la littérature. Comme antécédent prépondérant dans la perception du risque, on retient principalement : la sensibilité au risque spécifique, la culture du touriste, la nouveauté perçue, l'expérience négative, les réactions affectives suscitées par le choix et la difficulté perçue du choix. En partant des résultats de l'étude qualitative et compte tenu de la revue de la littérature, nous retiendrons neuf facteurs à l'origine du risque perçu dans le tourisme. Six facteurs ont été révélés par des recherches antérieures, soit : la sensibilité au risque spécifique, la culture, la nouveauté perçue, l'expérience négative, les réactions affectives négatives suscitées par le choix et la difficulté perçue du choix. L'étude qualitative a fait émerger trois nouveaux facteurs : la sensibilité au coût du séjour, l'insécurité perçue ainsi que l'exposition au web et aux réseaux sociaux.

En se fondant sur les approches de Bauer (1960), Mallet (2004) ou Ayadi (2006) et sur le paradigme de Volle (1995), les neuf facteurs à l'origine du risque perçu dans le choix d'une destination touristique peuvent être regroupés en trois catégories. La première catégorie 
concerne les caractéristiques psychographiques du touriste et regroupe « la sensibilité au risque spécifique », "la sensibilité au coût du séjour » et

" la culture du touriste ». La deuxième catégorie concerne les caractéristiques perçues de la destination touristique et regroupe : " la nouveauté perçue ", "l'expérience négative » et " l'insécurité perçue de la destination touristique ». Enfin, la troisième catégorie concerne les caractéristiques subjectives du choix d'un voyage touristique et regroupe " les réactions affectives négatives suscitées par le choix » comprenant l'anxiété, le souci, le stress et la nervosité, "l'influence des médias » dont l'exposition au web et aux réseaux sociaux, ainsi que " la difficulté perçue du choix d'une destination touristique ». Ces trois catégories sont représentées schématiquement dans le modèle conceptuel (figure 1).

Figure 1 : Modèle conceptuel des antécédents du risque perçu dans le choix d'une destination touristique

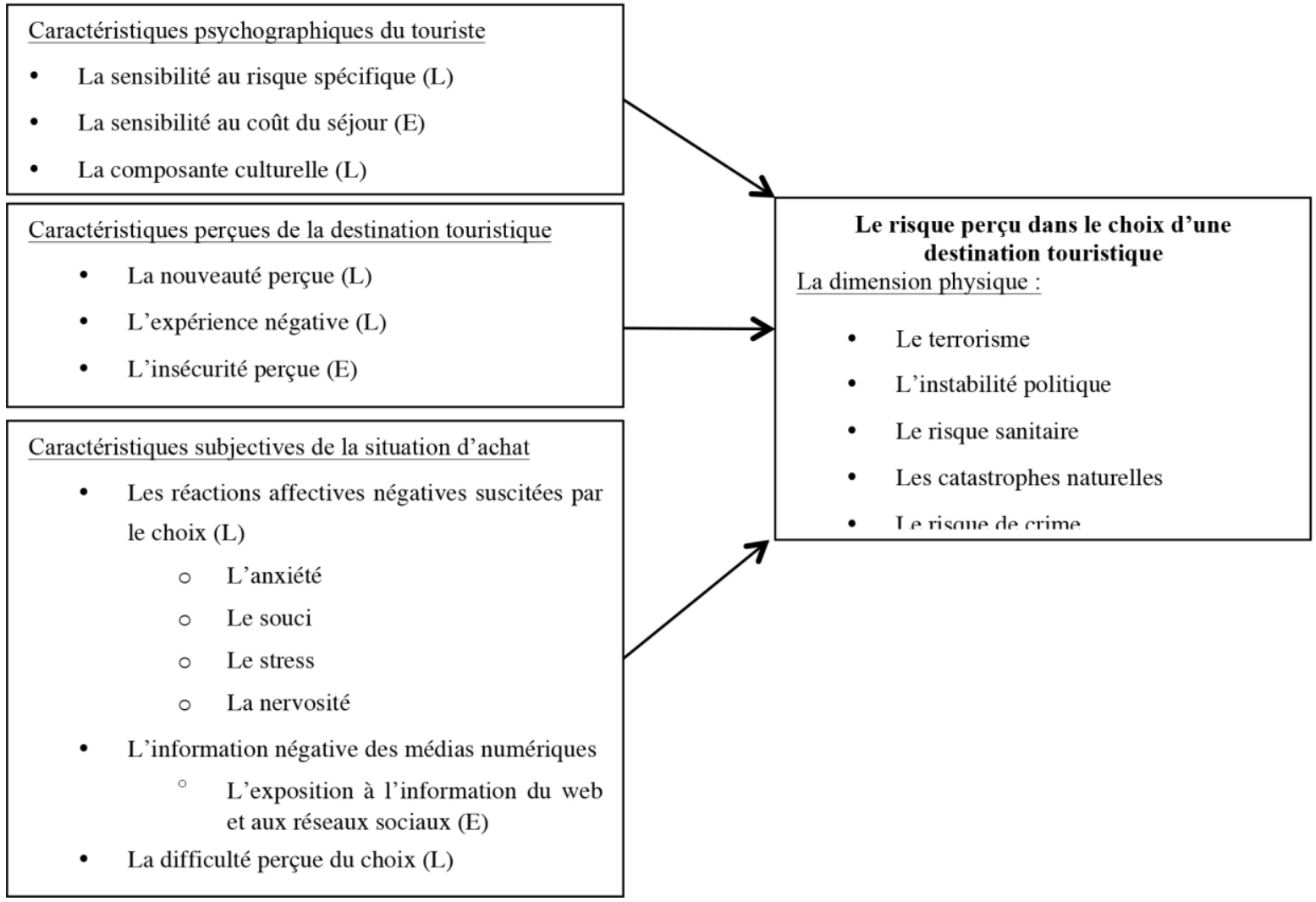

\subsection{Les dimensions du risque perçu dans le tourisme}

Parmi les risques perçus dans le tourisme identifiés dans la littérature académique, nos interlocuteurs en soulignent cinq : le terrorisme, l'instabilité politique, le risque sanitaire, le crime et les catastrophes naturelles, qui sont surtout liés à la dimension physique. La figure 1 présente un modèle conceptuel qui lie les facteurs subjectifs à l'origine du risque perçu dans le choix d'une destination touristique, à la dimension physique de ce risque. On représente par $(L)$ les facteurs identifiés dans la littérature et $(E)$ les antécédents qui ont émergé de l'étude qualitative exploratoire.

\section{Discussion des résultats}

L'objectif de notre étude est d'enrichir la connaissance sur le risque perçu lors du choix d'une destination touristique. La figure 1 regroupe neuf facteurs subjectifs qui sont à l'origine de la perception d'un risque par un touriste. Sur la base conjointe de la revue de littérature et nos 
entretiens qualitatifs avec des experts du tourisme, le tableau 3 approfondit les neuf antécédents du risque perçu dans le choix d'une destination touristique.

Tableau 3 : Les neuf facteurs à l'origine du risque perçu dans le tourisme

\begin{tabular}{|c|c|}
\hline $\begin{array}{l}\text { La } \\
\text { sensibilité } \\
\text { au risque } \\
\text { spécifique }\end{array}$ & $\begin{array}{l}\text { Mallet (2004) distingue deux types de sensibilité au risque, à savoir la sensibilité au } \\
\text { risque global et la sensibilité au risque spécifique. Notre étude qualitative confirme } \\
\text { l'importance de la sensibilité au risque spécifique dans l'émergence du risque perçu. Les } \\
\text { experts affirment que les touristes peuvent être préoccupés quant aux différents risques } \\
\text { spécifiques qui peuvent mettre en péril un séjour touristique. }\end{array}$ \\
\hline $\begin{array}{l}\text { La } \\
\text { sensibilité } \\
\text { au coûtdu } \\
\text { séjour }\end{array}$ & $\begin{array}{l}\text { Le facteur sensibilité au coût du séjour a émergé des entretiens semi-directifs. Les } \\
\text { professionnels du tourisme estiment que les touristes sont sensibles au coût impliqué } \\
\text { par le choix d'un voyage touristique, et ce quel que soit son niveau. «Le touriste perçoit } \\
\text { un risque dans le cas d'un séjour ayant un coût élevé et dans le cas d'un séjour à moindre } \\
\text { coût ». Pour compléter ces propos, Tare Sayed (2010) montre que la baisse du prix du } \\
\text { voyage vers une destination touristique est corrélée positivement avec le risque perçu. Les } \\
\text { touristes français associent par exemple la baisse du prix de la destination Égypte à une } \\
\text { mauvaise qualité du séjour ou du service et àl'insécurité du pays. Contrairement à cela, un } \\
\text { prixélevéengendre un risque perçu, notammentfinancier. S'agissantd'une caractéristique } \\
\text { psychologique et individuelle, cette sensibilité est différente d'un touriste à l'autre. }\end{array}$ \\
\hline $\begin{array}{l}\text { La culture } \\
\text { du touriste }\end{array}$ & $\begin{array}{l}\text { L'opinion des professionnels du tourisme sur la question de la culture du touriste et son } \\
\text { influence sur la perception du risque diverge. D'une part, les différences culturelles sont } \\
\text { perçues comme étant un vecteur d'attractivité et de richesse de la destination. C'est un } \\
\text { élément indispensable dans la commercialisation du produit touristique. Les intermédiaires } \\
\text { du tourisme tentent de convertir les divergences culturelles en une véritable expérience } \\
\text { d'apprentissage. À contrario, le choc culturel peut avoir des incidences négatives sur le } \\
\text { touriste. Les incompatibilités sociales peuvent créer une crainte et une réticence à l'idée } \\
\text { du voyage et de la rencontre de l'autre. }\end{array}$ \\
\hline \begin{tabular}{l}
\multicolumn{1}{c}{ La } \\
nouveauté \\
perçue de la \\
destination \\
touristique
\end{tabular} & $\begin{array}{l}\text { Les travaux de Popielarz (1967) montrent une relation positive entre le risque perçu et } \\
\text { la nouveauté perçue d'un produit. Crompton (1979) confirme que la nouveauté est l'un } \\
\text { des facteurs qui influencent le choix de la destination touristique. Selon Lepp et Gibson } \\
\text { (2003), les destinations perçues comme nouvelles suscitent plus de risque perçu que les } \\
\text { destinations auxquelles le touriste est habitué. Cependant, ils affirment aussi que dans le } \\
\text { tourisme, les chercheurs exploitent moins la nouveauté comme un facteur de risque perçu } \\
\text { et de contrainte que comme un facteur de motivation et de découverte. Nos entretiens } \\
\text { qualitatifs confirment cette idée. La nouveauté perçue dans le tourisme ne concerne } \\
\text { pas uniquement les “nouvelles destinations touristiques", mais traduisent aussi une } \\
\text { nouvelle façon de voyager, de réserver un séjour ou autre nouveau concept touristique } \\
\text { Nos interlocuteurs constatent aussi que les touristes optent souvent pour « les pratiques } \\
\text { familières » au lieu de s'intéresser aux nouvelles formes du tourisme. }\end{array}$ \\
\hline
\end{tabular}




\begin{tabular}{|c|c|}
\hline $\begin{array}{l}\text { L'expérience } \\
\text { négative }\end{array}$ & $\begin{array}{l}\text { Lepp et Gibson (2003) puis Tare Sayed (2010) stipulent qu'un touriste perçoit moins } \\
\text { de risque lors du choix d'une destination dans laquelle il s'est déjà rendu. Pour } \\
\text { Mallet (2004), l'expérience négative est une source du risque perçu. Les résultats } \\
\text { de notre enquête auprès des professionnels du tourisme se rapprochent davantage } \\
\text { des conclusions de Mallet (2004). De plus, la littérature nous apprend qu'un } \\
\text { consommateur ayant déjà été confronté à une expérience négative liée à l'achat ou à } \\
\text { l'usage d'un produit ou d'un service percevrait une plus grande probabilité que cette } \\
\text { conséquence se produise dans de futurs achats similaires (Cunningham, 1967). }\end{array}$ \\
\hline $\begin{array}{l}\text { L'insécurité } \\
\text { perçue } \\
\text { de la } \\
\text { destination } \\
\text { touristique }\end{array}$ & $\begin{array}{l}\text { La variable « insécurité perçue de la destination touristique » a émergé des } \\
\text { entretiens semi-directifs. Les études sur la sécurité dans le tourisme ont connu une } \\
\text { croissance exponentielle depuis les événements du } 11 \text { septembre } 2001 \text {. La } \\
\text { question de la sécurité s'est alors posée à plusieurs niveaux, y compris la sécurité } \\
\text { aéroportuaire, la sécurité des procédures aériennes, la sécurité des clubs de } \\
\text { vacances, des résidences de tourisme, des hôtels, de la qualité alimentaire des } \\
\text { restaurants, etc. Face à ce besoin de sécurité de plus en plus indispensable pour le } \\
\text { touriste, les professionnels ont dû s'adapter en l'intégrant dans leurs offres } \\
\text { commerciales. D'après une étude IPSOS (2013), } 69 \% \text { des touristes ont une opinion } \\
\text { positive du développement des assurances voyage, de l'assistance rapatriement et } \\
\text { de l'ensemble des mesures qui permettent d'assurer la sécurité. La sécurité du } \\
\text { touriste ne concerne plus uniquement les prestataires du tourisme, mais cela devient } \\
\text { également une des préoccupations de l'ensemble des acteurs du secteur. }\end{array}$ \\
\hline $\begin{array}{l}\text { Les } \\
\text { réactions } \\
\text { affectives } \\
\text { négatives } \\
\text { suscitées } \\
\text { par le } \\
\text { choix de la } \\
\text { destination } \\
\text { touristique }\end{array}$ & $\begin{array}{l}\text { Des facteurs comme l'humeur et l'émotion sont considérés comme significativement } \\
\text { corrélés au risque perçu. Face à une situation d'achat, un consommateur de bonne } \\
\text { humeur perçoit moins de risque qu'un consommateur de mauvaise humeur (Volle, } \\
\text { 1995). Les résultats de Chaudhuri (1998) confortent les conclusions précédentes et } \\
\text { confirment l'influence positive des réactions affectives négatives sur le risque perçu. } \\
\text { Schaninger (1976) a également montré que l'anxiété en tant que trait de la } \\
\text { personnalité est corrélée positivement avec le risque perçu. Les seize entretiens } \\
\text { semi-directifs ont relevé l'importance de la dimension affective lors du choix d'un } \\
\text { séjour touristique. Elle est considérée comme étant un antécédent puissantdu risque } \\
\text { perçu lors du choix d'une destination. }\end{array}$ \\
\hline $\begin{array}{l}\text { L'influence } \\
\text { des médias } \\
\text { numériques }\end{array}$ & $\begin{array}{l}\text { L'influence des médias sur le risque perçu a suscité l'intérêt des chercheurs en } \\
\text { gestion. Combs et Slovic (1979) explorent le lien de causalité entre l'exposition aux } \\
\text { informations des médias et risque perçu. Les événements négatifs rapportés par } \\
\text { les médias sont surestimés par le consommateur, par exemple le crash d'avion, } \\
\text { contrairement aux événements non médiatisés comme l'accident de moto ou } \\
\text { de voiture dont la fréquence est pourtant souvent supérieure. L'environnement } \\
\text { informationnel a été considéré par Volle (1995) comme étant un antécédent du risque } \\
\text { perçu. Dans le domaine du tourisme, les entretiens qualitatifs soulignent l'importance } \\
\text { de l'information et du média consulté sur l'émergence du risque perçu. De plus, les } \\
\text { professionnels du tourisme pointent du doigt le rôle du web et des différents réseaux } \\
\text { sociaux dans la propagation des informations négatives qui suscitent le risque. }\end{array}$ \\
\hline $\begin{array}{l}\text { La difficulté } \\
\text { perçue du } \\
\text { choix de la } \\
\text { destination } \\
\text { touristique }\end{array}$ & $\begin{array}{l}\text { La difficulté perçue d'un choix se traduit par l'effort que le consommateur doit fournir } \\
\text { pour choisir un produit ou un service. Chaque catégorie de produit nécessite un } \\
\text { niveau d'effort différent. Mallet (2004) stipule que cette difficulté perçue du choix est } \\
\text { à l'origine du risque perçu pour des produits de nature complexe et durable. Dans le } \\
\text { tourisme, l'effort fourni dans le choix d'une destination est dû à la multitude d'offres } \\
\text { et de thématiques de voyage. Selon les professionnels du tourisme, les touristes } \\
\text { ont une réelle difficulté à choisir leur destination touristique et cela peut créer une } \\
\text { incertitude. }\end{array}$ \\
\hline
\end{tabular}




\section{CONCLUSION}

Notre recherche a précisé les principauxantécédents subjectifs du risque perçu dans le choix d'une destination touristique. Une multitude de facteurs a été identifiée par la littérature. Au total, on ne recense pas moins de dix-neuf variables provenant de trois sources différentes : les caractéristiques du consommateur, du produit et de la situation d'achat. Compte tenu de l'absence des études sur les déterminants du risque perçu lors du choix d'une destination touristique, nous avons mené seize entretienssemi-directifs avec les professionnels du tourisme dans la région Aquitaine. Au niveau de l'origine, nous avons confirmé que le risque perçu est la résultante de l'interaction de trois facteurs. D'abord, un touriste, caractérisé par sa culture, sa sensibilité au risque spécifique et sa sensibilité au coût du séjour. Ensuite, il a une perception de la destination touristique, en termes de nouveauté et d'insécurité et il a tendance à être influencé par une expérience négative d'un séjour touristique. Enfin, le touriste vit une situation d'achat subjective en termes de réactions affectives négatives suscitées par le choix, de la difficulté perçue du choix et de l'influence des informations relayées par les média et réseaux sociaux.

Malgré le biais généré par la perception indirecte des professionnels du choix d'un touristeconsommateur et leur interprétation de la perception du risque, notre étude qualitative apporte de nouvelles connaissances et enrichit la littérature. Elle a permis l'émergence de trois nouveaux déterminants subjectifs du risque perçu dans le tourisme : la sensibilité au coût du séjour, l'insécurité perçue et l'exposition du touriste à l'information du web et des réseaux sociaux. Elle a également permis un approfondissement de la compréhension des variables identifiées dans la littérature. Enfin, nous avons démontré que le risque perçu dans le tourisme est opérationnalisé par cinq dimensions du risque physique, à savoir : le terrorisme, l'instabilité politique, le risque sanitaire, le crime et les catastrophes naturelles.

Cette étude contribue au renouvellement de la recherche sur les antécédents du risque perçu. Afin de pallier l'absence de généralisation, qui n'estpas permise parl'étude qualitative exploratoire, et dans le but d'atténuer le biais que pose l'interrogation des professionnels du tourisme, une analyse confirmatoire sur un échantillon représentatif de touristes français, à travers un questionnaire est en cours de réalisation. Le but est de quantifier le modèle conceptuel ce qui permet d'hiérarchiser l'importance et l'interaction des différents antécédents du risque perçu dans le tourisme. 


\section{BIBLIOGRAPHIE}

Ayadi N. (2010), L'expérience de prise de risque par les consommateurs : rôle des émotions anticipées positives, Recherche et Applications en marketing, vol.25, $n^{\circ} 2$.

Ayadi N. (2006), Étude du Comportement du Consommateur Face au Risque : Proposition d'un Modèle Expérientiel, Actes du 5e Congrès International des Tendances du Marketing, Venise, Italie, 20 et 21 janvier.

Bardin L. (1977). L'analyse du contenu, Presses Universitaires de France.

Bauer R.A. (1960), Consumer Behavior as Risk Taking, dans Robert S. Hancock, Dynamic Marketing for a Changing World, Chicago.

Brunel O. et Pichon P.E. (2002), Proposition d'une Classification des Réducteurs de Risque Alimentaire Intégrant l'Acte d'Achat et de consommation. Actes du Congrès International de l'Association Française de Marketing, Lille.

Calandre N. (2006), Pratiques et perception des risques nutritionnels : les mères face aux malnutritions infantiles au Vietnam, Thèse de Doctorat en gestion du développement agricole, agroalimentaire et rural, Université de Montpellier 1.

Cases A.S. (2001), La réduction du risque dans le contexte de l'achat électronique sur internet, Thèse de Doctorat en Sciences de Gestion, Université de Montpellier II.

Chaudhuri A. (1998), Product Class Effects on Perceived Risk : The Role Of Emotion, International journal of research in marketing, vol.15, $n^{\circ} 2$.

Combs B. \& Slovic P. (1979), Newspaper Coverage of Causes of Death, Journalism Quarterly, vol.56, n4.

Crompton J.L. (1979), Motivations For Pleasure Vacation, Annals Of Tourism Research, vol. $6, n^{\circ} 4$.

Croutsche J.J et Roux M. (2005), Risques et tourisme : vers un modèle causal d'évaluation de l'impact des risques sur le comportement des touristes, Téoros, vol.24, $\mathrm{n}^{\circ} 1$.

Cunningham S. (1967), The Major Dimensions of Perceived Risk, dans Cox D., Risk Taking and Information Handling in Consumer Behavior, Harvard University Press.

Easterby-Smith M., Thorpe, R. et Jackson P. (2008), Management Research, 3rd éd., SAGE

Korstanje M.E. (2011), The fear of Traveling : a New Perspective For Tourism And Hospitality, An International Journal of Tourism and Hospitality Research, vol.22, n`2.

Lepp A. \& Gibson H. (2003), Tourist roles, perceived risk and international tourism, Annals of Tourism Research, vol. $30, n^{\circ} 3$.

Mallet S. (2004), À la Recherche des Origines du Risque Perçu à l'Égard de l'Achat d'un Produit, La troisième journée normande de la consommation, Rouen, 11 et 12 mars.

Miles M.B. \& Huberman A.M. (1994), Qualitative Data Analysis : An Expanded Sourcebook, Sage.

Mitchell V.W. \& Vassos V. (1997), Perceived Risk and Risk Reduction in Holiday Purchases: A CrossCultural and Gender Analysis, Journal of Euromarketing, vol.6, $n^{\circ} 3$.

Peter J.P. \& Ryan M.J. (1976), An Investigation of Perceived Risk at the Brand Level, Journal of Marketing Research, vol.13.

Popielarz D.T. (1967), An Exploration of Perceived Risk and Willigness To Try New Products, Journal of Marketing Research, vol.4, $\mathrm{n}^{\circ} 4$.

Raju P.S. (1980), Optimum Stimulation Level : Its Relationship to Personality, Demographics, and Exploratory Behavior, Journal Of Consumer Research, vol.7, $n^{\circ} 3$.

Rippl S. (2002), Cultural Theory and Risk Perception : A Proposal For a Better Measurement, Journal of risk research, vol.5, $\mathrm{n}^{\circ} 2$.

Roehl W. \& Fesenmaier D. (1992), Risk Perceptions and Pleasure Travel : An Exploratory Analysis, Journal of Travel Research, vol. 30, n4. 
Schaninger C.M. (1976), Perceived Risk and Personality, Journal of Consumer Research, vol. 3, $\mathrm{n}^{\circ} 2$.

Sjöberg L. (2000), Factors in Risk Perception, Risk Analysis, vol. 20, nº 1.

Sönmez S.F. (1998), Tourism, Terrorism, and Political Instability, Annals of Tourism Research, vol.25, $n^{\circ} 2$.

Sönmez S.F. \& Graefea R. (1998), Influence of Terrorism Risk on Foreign Tourism Decisions, Annals of Tourism Research, vol.25, n¹.

Stone R.N. \& Gronhaug K. (1993), Perceived Risk : Further Considerations for the Marketing Discipline, European Journal of Marketing, vol.27, n`3.

Tare Sayed A.A. (2010), The Relationship Between the Perception of Risk and the Decision Making Process of Travel of French Tourist : The Case of Egypt, An International Multidisciplinary Journal of Tourism, vol.5, $\mathrm{n}^{\circ} 2$.

Taylor J. (1974), The Role of Risk in Consumer Behaviour, Journal of Marketing, vol.38, n`2.

Verhage B.J., Yavas U. \& Green R.T. (1991), Perceived Risk : A Cross-Cultural Phenomenon? International Journal of Research in Marketing, vol.7, $\mathrm{n}^{\circ} 4$.

Volle P. (1995), Le concept du risque perçu en psychologie du consommateur, Revue Recherche et Applications en Marketing, vol.10, $\mathrm{n}^{\circ} 1$. 